\title{
Conventional and Diffusion-Weighted MR Imaging Findings of Parotid Gland Tumors
}

\author{
Original Investigation $>$ Fakih Cihat Eravc1 ${ }^{1}\left(\mathbb{D}\right.$, Deniz Sözmen Cllız $^{2}$ (D), Kürşat Murat Özcan ${ }^{1}$ (D), Mustafa Çolak ${ }^{1}$ (D), \\ Mehtap Çavuşoğlu ${ }^{2}$ (D), Süleyman Emre Karakurt ${ }^{1}$ (D), Mehmet Fatih Karakuş ${ }^{1}$ (1) \\ ${ }^{1}$ Department of Otorhinolaryngology, University of Health Sciences, Ankara Numune Training and Research Hospital, Ankara, Turkey \\ ${ }^{2}$ Department of Radiology, University of Health Sciences, Ankara Numune Training and Research Hospital, Ankara, Turkey
}

Abstract

ORCID iDs of the authors: F.C.E. 0000-0001-9092-7923; D.S.C. 0000-0003-3640-9273; K.M. $0.0000-0002-5262-0565$ M.C. 0000-0002-3191-4134; M. Çavusoğlu 0000-0003-3966-1415; S.E.K. 0000-0002-3394-8119; M.F.K. 0000-0002-6264-5416.

Cite this article as: Erava FC, Sözmen CIIIz D, Özcan KM, Colak M, Ģavusoğlu M, Karakurt SE, et al. Conventional and Diffusion-Weighted MR Imaging Findings of Parotid Gland Tumors. Turk Arch Otorhinolaryngol 2020; 58(3): 174-80.

Corresponding Author:

Fakih Cihat Eravcl, feravci@gmail.com

Received Date: 11.04 .2020

Accepted Date: 31.05 .2020

Content of this journal is licensed under a Creative Commons Attribution 4.0 International License. Available online at www.turkarchotolaryngol.net
Objective: To investigate diffusion-weighted magnetic resonance imaging (MRI) findings of parotid gland lesions in addition to conventional MRI findings and demographic data.

Methods: A retrospective evaluation was made of the demographic data, histopathologic data, preoperative conventional and diffusion-weighted MRI of 74 patients who underwent parotidectomy. The patients were categorized according to the histopathology (pleomorphic adenoma [PA], Warthin's Tumor [WT] and malignant Tumor [MT]).

Results: Histologically, 30 patients had PA, 27 patients had WT, and the remaining 17 patients had MT. The mean age of the PA, WT and MT groups were $44 \pm 21(20-72), 55 \pm 10(41-71)$ and $62 \pm 20(21$ 76) years, respectively. The WT (81\%) and MT (70\%) groups were male dominant, while the PA group showed female dominance (55\%). The PA group showed statistically significant difference in terms of age $(p<0.05)$ and gender $(p=0.009)$ compared to the other two groups. The median apparent diffusion coefficient (ADC) values for the PA, WT and MT groups were $1.99 \pm 0.94(1.10-2.41) \times 10-3 \mathrm{~mm}^{2} / \mathrm{s}$, $0.92 \pm 0.35(0.21-1.79) \times 10-3 \mathrm{~mm}^{2} / \mathrm{s}$ and $1.20 \pm 0.34$ $(0.78-1.47) \times 10-3 \mathrm{~mm}^{2} / \mathrm{s}$, respectively. PA was differentiated from the other two groups $(p=0.001)$. The sensitivity and specificity for distinguishing PAs from WT was $97 \%$ and $85 \%$, respectively, when the ADC cutoff value was 1.25; and for distinguishing PAs from MT was $77 \%$ and $83 \%$, respectively, when the ADC cutoff value was 1.35 .

Conclusion: ADC measurements are useful for the differentiation of PA from both WT and MT; and can be used as a complementary tool to predict the histopathology in the preoperative planning of parotid tumors.

Keywords: Parotid neoplasms, magnetic resonance imaging, diffusion-weighted imaging, apparent diffusion coefficient, pleomorphic adenoma

\section{Introduction}

Salivary gland tumors constitute about 3-6\% of all head and neck tumors, and $80 \%$ are located in the parotid gland (1). Parotid gland tumors are predominantly benign tumors and the most common type is pleomorphic adenoma (PA). Approximately a quarter (15-32\%) of the cases show malignant histology (2). The standard treatment of parotid tumors is surgical excision, and in most cases superficial parotidectomy is sufficient. Warthin's tumor (WT), which is the second most common benign parotid gland tumor, can be approached more conservatively as the incidence of malignant transformation is low. However, total parotidectomy and additional elective neck dissection may be required in parotid tumors with deep lobe localization and high-grade malignant tumors (MT). Therefore, whether the histopathology is malignant or benign is particularly important in preoperative planning of the surgery. In addition, considering the difficulty and relatively lower facial protection rates of revision-complementing total parotidectomies, preoperative information about the histopathology is critical to avoid morbidities.

Patient demographic information and some clinical features of the parotid mass contribute to the malignant-benign distinction. Pain, facial paralysis, rapid growth, skin infiltration and irregular boundaries suggest malignancy (2), whereas an 
older age, smoking history, and male gender increase the probability of a Warthin's benign lesion (3). In addition to this clinical information, preoperative imaging provides valuable information for surgical planning, such as the location of the mass and its relationship with vital structures (4). Ultrasonography (USG) guided fine needle aspiration biopsy (FNAB) is the most commonly used imaging method with high diagnosis rates (81-98\% in benign and malignant distinction) $(5,6)$. There is, however, a risk of tumor seeding during this intervention, which might increase the risk of recurrence (7). In addition, insufficient sampling because of the heterogeneity of the lesions and the need for an experienced cytologist to detect specific histopathology, increases false positive or false negative rates. Furthermore, the inability to evaluate deep structures and operator-dependence are the other shortcomings of USG. Another imaging modality is computed tomography (CT), but this is used less commonly than X-rays and has contrast-related side effects (8).

The use of magnetic resonance imaging (MRI) has become more prevalent with increased availability, and with technological advancements it now contributes substantially to preoperative planning. Despite the disadvantages of the above-mentioned modalities, MRI is non-invasive and does not expose the patient to radiation. Most importantly, it provides information about the location of the mass, its relationship with vital structures and the presence of accompanying lymphadenopathy in the neck. Recent studies have shown that different parameters in MRI can be used for benign - malignant differentiation (9-11). In this context, the contribution of conventional findings such as lesion morphology, signal intensity, enhancement pattern has been investigated (4). It has also been stated that apparent diffusion coefficient (ADC) measurements obtained with diffusion-weighted imaging (DWI), which provides quantitative information about the microcirculation and vascularization properties of the mass, can contribute to this differentiation (10-13). It also has the important advantage that this measurement can be carried out practically without the need for contrast agents. However, there is a controversy related to ADC values where some found it useful for benign malignant differentiation while others found an overlap (14). Therefore, the aim of this study

\section{Main Points}

- In parotid lesions, preoperative histopathology information is critical in terms of planning the extent of the surgery and avoiding morbidity

- MRI is a non-invasive modality which can be used for preoperative benign - malignant differentiation.

- In addition to the conventional findings (such as lesion morphology, signal intensity, enhancement pattern), apparent diffusion coefficient (ADC) measurements obtained with diffusion-weighted imaging (DWI) reveals promising information.

- The results of our study showed that pleomorphic adenoma (PA) can be accurately differentiated from malignant and Warthin's tumors according to ADC values.

- ADC measurements can prevent unnecessary biopsies in the majority of parotid tumors since the most common type of parotid tumor seen is $\mathrm{PA}$. was to investigate the relationship between the conventional signal intensity level and ADC values on MRI with the histopathological results of the parotid gland operations which were performed in our clinic in a five-year period. This may provide an opportunity to predict the parotid masses without the need of invasive interventions.

\section{Methods}

From the surgical archives, 142 patients were identified who underwent surgery in our department for a parotid mass in the years 2014 through 2018, and of these, those with preoperative MR images were included in the study. Patients were excluded from the study if there was no MRI examination (50 patients), if there was MRI examination but no diffusion-weighted imaging (DWI) sequences (eight patients), and if they had a diagnosis of recurrent parotid masses (six patients) or inflammatory lesions (two patients). Patients with lymphoma histopathology (two patients with high diffusion restriction) were not included in the statistical analyses in order to eliminate extreme values and obtain ADC homogeneity in the malignant histology group. Thus, retrospective evaluation was performed using the demographic data, the histopathologic data and the MRI results of the remaining 74 patients. The study was approved by the Clinical Research Ethics Committee of University of Health Sciences, Ankara Numune Training and Research Hospital (Approval Date: September 5, 2019; Approval Number: E1047-2019) and all procedures followed were in accordance with the Helsinki Declaration. Informed consent was preoperatively obtained from all participating subjects. Histopathological diagnoses were obtained from the surgical specimens and three study groups were defined according to the histopathologic examination results (pleomorphic adenoma, Warthin's Tumor and malignant tumor).

\section{MRI}

A 1.5 T MR unit (Excite, GE Medical Systems, Milwaukee, WI, USA) was used for MRI, and images were obtained with head and neck array coils. The gradient power was $33 \mathrm{mT} / \mathrm{s}$. In the evaluations, $\mathrm{T} 1$ - and $\mathrm{T} 2$-weighted axial sequences were used and features for this sequences were $500 \mathrm{~ms} \mathrm{TR}, 15.7 \mathrm{~ms} \mathrm{TE}$ for T1-weighted images, and $3000 \mathrm{~ms}$ TR, $104.8 \mathrm{~ms}$ TE for T2-weighted images. The mutual utilized features for both images were: $3 \mathrm{~mm}$ slice thickness, $0.5 \mathrm{~mm}$ inter-slice gap; $20 \times 20$ $\mathrm{cm}$ field of view, 320×224 matrix, 3 excitations. T2-weighted images were achieved with fast spin echo (FSE) sequences. Additionally, DWI sequence images $(10,000 \mathrm{~ms}$ repetition time, $85.8 \mathrm{~ms}$ echo time, $4 \mathrm{~mm}$ slice thickness, $1 \mathrm{~mm}$ inter-slice gap, $128 \times 128$ matrix) were performed with echo planar single shot spin echo imaging (b values $=0$ and $1000 \mathrm{~s} / \mathrm{mm}^{2}$ ). In order to obtain three sets of DWI diffusion gradients, three different orthogonal directions ( $\mathrm{x}, \mathrm{y}, \mathrm{z}$ axes) were performed. ADC measurement results were obtained automatically.

\section{Image Analyses}

ADC maps were obtained automatically from DWI sequences. ADC data were calculated through a circular region of interest $0.67-0.70 \mathrm{~cm}^{2}$. The mean measurement value was de- 
rived from two to six measurements for each lesion by both senior radiologists who did not know the histopathology. These measurements were executed at solid parts of the lesions where diffusion was restricted. If the mass did not have any area of diffusion restrictions, increased diffusion fields were used. In heterogeneous tumor masses, cystic necrotic portions were not considered, but the solid components were used for the measurement. In conventional MRI, lesion signal intensity on T1-weighted images was indicated as hypointense if the intensity level was equal to or lower than that of muscle intensity, and as hyperintense if the intensity level were brighter than that of the muscle. T2-weighted intensity was reported as hypointense if the intensity level was lower than that of the normal parotid tissue, and as hyperintense if the intensity level were equal to or brighter than that of the normal parotid tissue. The classification was made according to a part of the lesion $\geq 50 \%$ in heterogeneous lesions.

\section{Statistical Analysis}

Study data were analyzed statistically using IBM Statistical Package for Social Sciences version 22 software (IBM SPSS Inc.; Armonk, NY, USA). Conformity of the data to normal distribution was assessed using the Kolmogorov-Smirnov test. Continuous variables were presented as median \pm interquartile range and discrete variables as frequencies and percentages. The age and $\mathrm{ADC}$ values for each group (PAs, WTs and MTs) were compared with Kruskal-Wallis non-parametric analysis of variance and Tamhane's T2 test was used for multiple comparison. The inter- and intra-observer reproducibility for ADC maps measurements were evaluated using the intra-class correlation coefficient (ICC) to assess the degree of absolute agreement among ADC measurements. An ICC >0.80 was considered to be indicative of acceptable agreement. The gender analysis results of the groups were compared with the Pearson Chi square test. To determine the sensitivity and specificity of the images for the prediction of tumor histology, Receiver Operating Characteristic (ROC) analysis was applied. A value of $\mathrm{p}<0.05$ was considered statistically significant.

\section{Results}

The study group comprised 47 (63.5\%) male and 27 (36.5\%) female patients with a mean age of $51.8 \pm 12.9$ years (range, 20 76 years). Histologically, 30 patients had pleomorphic adenoma, 27 patients had Warthin's tumor, and the remaining 17 patients had malignant histopathology (five mucoepidermoid carcinoma, four malignant melanoma, two adenocarcinoma, two acinic cell carcinoma, one malignant epithelial tumor, one adenoid cystic carcinoma, one salivary duct carcinoma and one squamous cell carcinoma). The mean age and gender percentage for each group are shown in Table 1. The PA group was younger compared to

Table 1. Gender data and mean ages of the study groups

\begin{tabular}{|c|c|c|c|c|c|}
\hline & Male & Female & $\begin{array}{l}\text { Age (year) } \\
\text { Median } \pm \text { interquartile } \\
\text { range (min-max) }\end{array}$ & $\begin{array}{l}\text { Kruskal-Wallis } \\
\text { Test }\end{array}$ & $\begin{array}{l}\text { Tamhane's } \mathrm{T} 2 \\
\text { multiple comparison } \\
\text { test }\end{array}$ \\
\hline \multicolumn{6}{|c|}{ Histopathology (n) } \\
\hline PA (30) & $13(45 \%)$ & $17(55 \%)$ & $44 \pm 21(20-72)$ & \multirow{3}{*}{$\mathrm{p}=0.001$} & $(\mathrm{PA}-\mathrm{WT}) \mathrm{p}=0.001$ \\
\hline WT (27) & $22(81 \%)$ & $5(19 \%)$ & $55 \pm 10(41-71)$ & & $(\mathrm{PA}-\mathrm{MT}) \mathrm{p}=0.010$ \\
\hline MT (17) & $12(70 \%)$ & $5(30 \%)$ & $62 \pm 20(21-76)$ & & $(\mathrm{WT}-\mathrm{MT}) \mathrm{p}=0.973$ \\
\hline
\end{tabular}

min: minimum; max: maximum; PA: pleomorphic adenoma; WT: Warthin's tumor; MT: malignant tumor

Table 2. Intensity levels (hypointense and hyperintense) at T1- and T2-weighted images for each histopathology

\begin{tabular}{|c|c|c|c|c|}
\hline & \multicolumn{2}{|c|}{$\mathrm{T} 1$} & \multicolumn{2}{|c|}{$\mathrm{T} 2$} \\
\hline & Hypointense & Hyperintense & Hypointense & Hyperintense \\
\hline \multicolumn{5}{|l|}{ Histopathology (n) } \\
\hline $\mathrm{PA}(30)$ & $30(100 \%)$ & $0(0 \%)$ & $5(17 \%)$ & $25(83 \%)$ \\
\hline WT (27) & $18(66 \%)$ & $9(34 \%)$ & $22(81 \%)$ & $5(19 \%)$ \\
\hline MT (17) & $15(88 \%)$ & $2(12 \%)$ & $8(47 \%)$ & $9(53 \%)$ \\
\hline - Mucoepidermoid carcinoma (n:5) & 4 & 1 & 3 & 2 \\
\hline - Malignant melanoma (n:4) & 3 & 1 & 1 & 3 \\
\hline - Adenocarcinoma (n:2) & 2 & 0 & 1 & 1 \\
\hline - Acinic cell carcinoma (n:2) & 2 & 0 & 0 & 2 \\
\hline - Malignant epithelial tumor (n:1) & 1 & 0 & 0 & 1 \\
\hline - Adenoid cystic carcinoma (n:1) & 1 & 0 & 1 & 0 \\
\hline - Salivary duct carcinoma (n:1) & 1 & 0 & 1 & 0 \\
\hline - Squamous cell carcinoma (n:1) & 1 & 0 & 1 & 0 \\
\hline
\end{tabular}

PA: pleomorphic adenoma; WT: Warthin's tumor; MT: malignant tumor 
the other two groups ( $<<0.05$ for both groups) and female dominant $(\mathrm{p}=0.009)$.

The intensity levels on conventional MRI T1- and T2-weighted images for each of the histological types are tabulated in Table 2. The PA, WT and MT groups of tumors were predominantly hypointense $(100 \%, 66 \%$ and $88 \%$, respectively) on T1-weighted images. On T2-weighted images, PAs were hyperintense (83\%), and WTs were predominantly hypointense (81\%). Unlike these benign lesions, the number of MTs on T2-weighted images were nearly equal (47\% hypointense and $53 \%$ hyperintense).

Intra- and interobserver agreements between the two radiologists were obtained for ADC values with mean ICCs of 0.946 and 0.882 , respectively. The ADC values on DWI for the PA, WT and MT groups are shown in Table 3, with the differentiation of PA from the other two groups $(\mathrm{p}=0.001)$. WT and MT were similar in terms of the mean ADC value $(\mathrm{p}=0.111)$. The lowest ADC value among the 74 patients was $0.21 \times 10^{-3}$ $\mathrm{mm}^{2} / \mathrm{s}$, seen in a case with WT. The lowest ADC values for MT was seen in acinic cell carcinoma $[0.95 \pm 0.35(0.78-1.13)] \times 10^{-3}$ $\mathrm{mm}^{2} / \mathrm{s}$. The two cases of lymphoma showed the lowest ADC values among malignant tumors $[0.41 \pm 0.26(0.22-0.60)] \times 10^{-3}$ $\mathrm{mm}^{2} / \mathrm{s}$ and therefore excluded from the analyses to make the malignant group as homogenous as possible.

The ROC analyses for the ADC values are shown in Figure 1. The area under the curve (AUC) was calculated as 0.948 and 0.927 , respectively, for the differentiation of PA from WT

Table 3. Mean ADC values for each group and histopathology

\begin{tabular}{l|l|l}
$\begin{array}{l}\text { ADC values }\left(\times 10^{-3} \mathrm{~mm}^{2} / \mathrm{s}\right) \\
\text { Mediantinterquartile range } \\
\text { (min-max) }\end{array}$ & $\begin{array}{l}\text { Kruskal-Wallis } \\
\text { Test }\end{array}$ & $\begin{array}{l}\text { Tamhane's T2 multiple } \\
\text { comparison test }\end{array}$ \\
\hline
\end{tabular}

Histopathology (n)

\begin{tabular}{|c|c|c|c|}
\hline $\mathrm{PA}(30)$ & $1.99 \pm 0.94(1.10-2.41)$ & \multirow{9}{*}{$\mathrm{p}=0.001$} & $(\mathrm{PA}-\mathrm{WT}) \mathrm{p}=0.001$ \\
\hline WT (27) & $0.92 \pm 0.35(0.21-1.79)$ & & $(\mathrm{PA}-\mathrm{MT}) \mathrm{p}=0.001$ \\
\hline MT (17) & $1.20 \pm 0.34(0.78-1.47)$ & & $(\mathrm{WT}-\mathrm{MT}) \mathrm{p}=0.111$ \\
\hline - Malignant melanoma (n:4) & $1.16 \pm 0.41(0.98-1.40)$ & & \\
\hline - Adenocarcinoma (n:2) & $1.12 \pm 0.39(0.85-1.40)$ & & \\
\hline - Malignant epithelial tumor (n:1) & 1.47 & & \\
\hline - Adenoid cystic carcinoma (n:1) & 1.00 & & \\
\hline - Salivary duct carcinoma (n:1) & 1.21 & & \\
\hline - Squamous cell carcinoma (n:1) & 1.26 & & \\
\hline
\end{tabular}

ADC: apparent diffusion coefficient; min: minimum; max: maximum; PA: pleomorphic adenoma; WT: Warthin's tumor; MT: malignant tumor

a

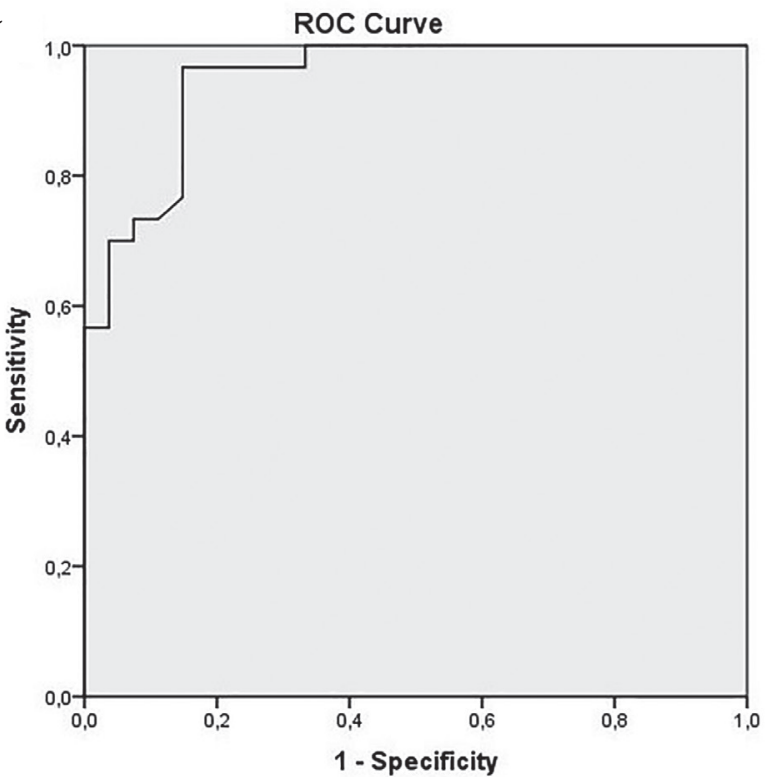

b

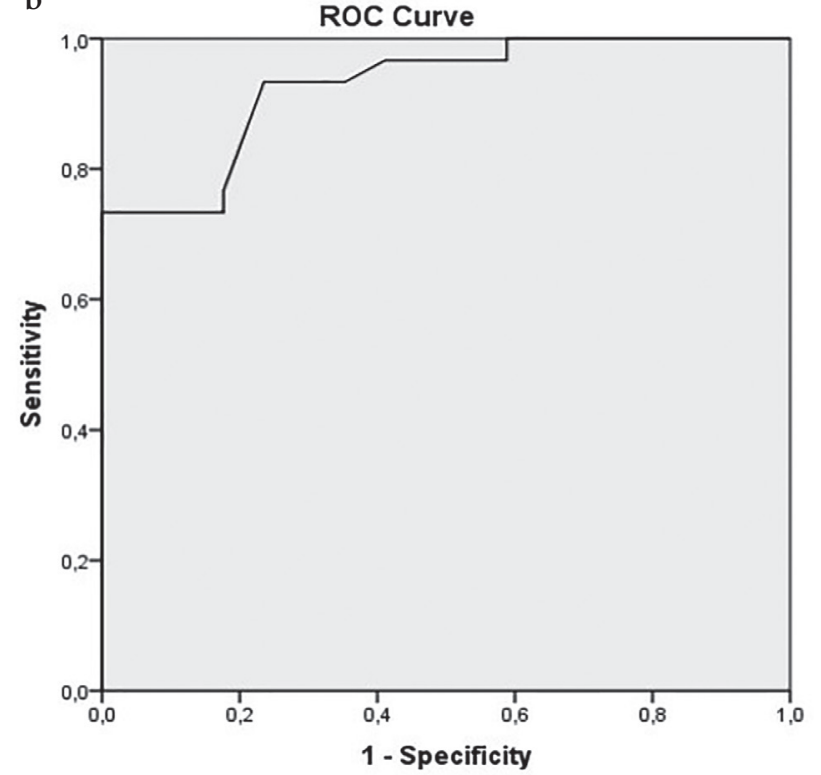



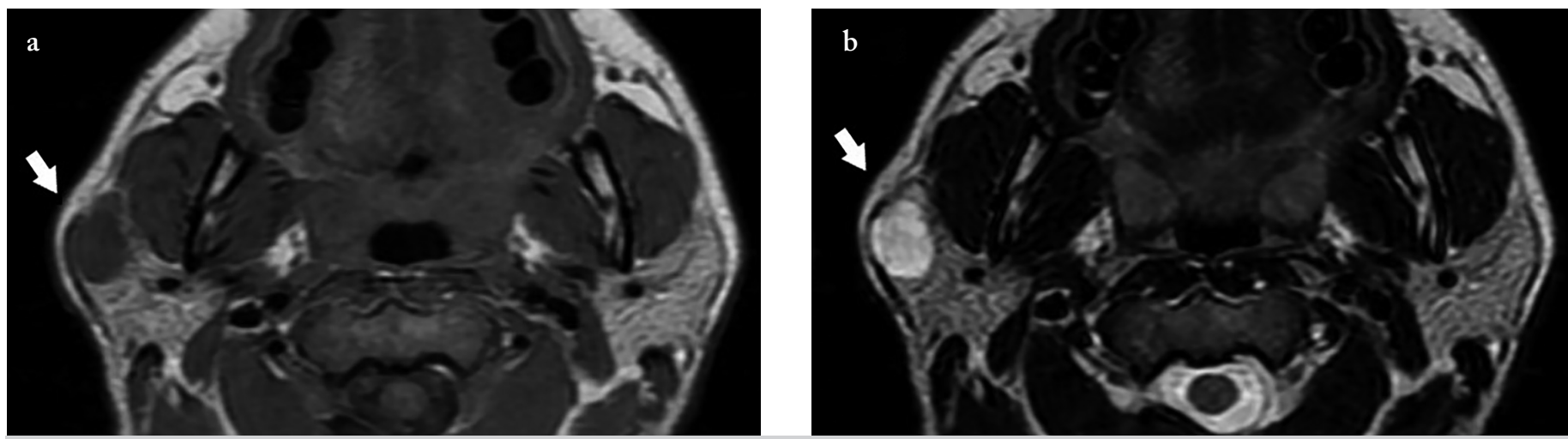

Figure 2. a, b. Axial MRI images from a 48-year-old patient with histologically proven PA in the right parotid gland (white arrows). (a) Hypointense signal intensity of the lesion in T1-weighted images, (b) hyperintense signal intensity of the lesion in T2-weighted images
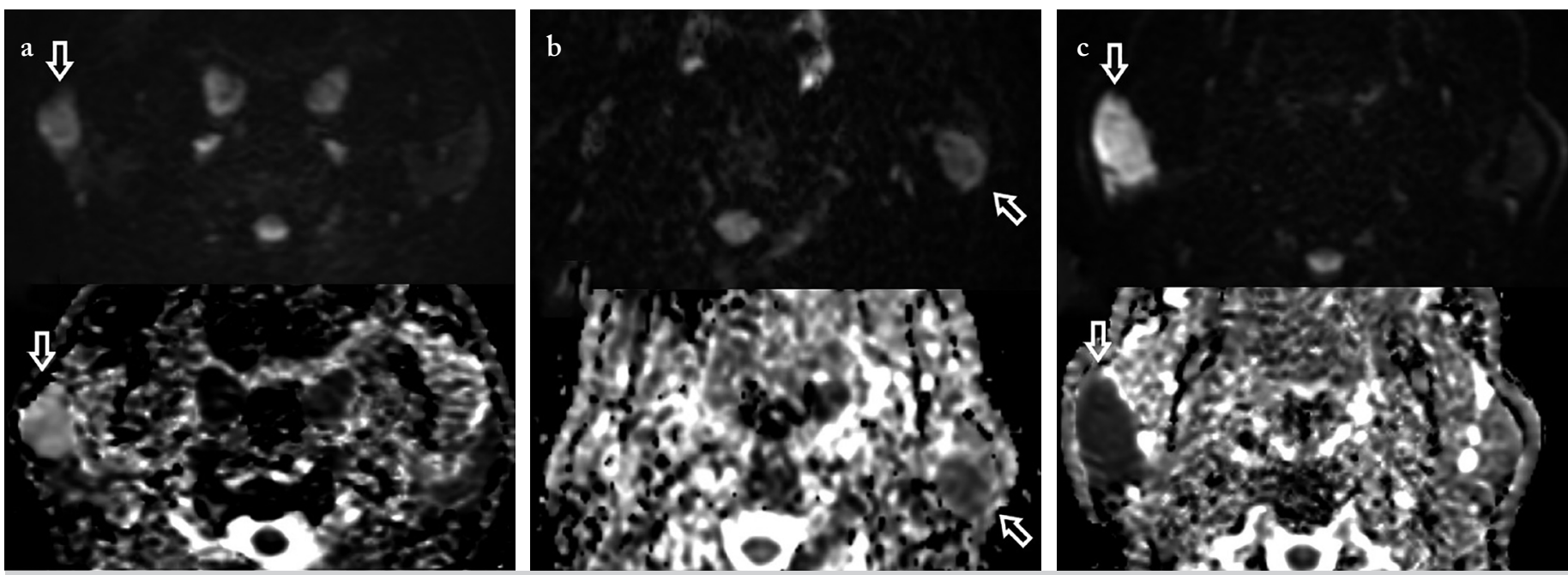

Figure 3. a-c. The lesions (arrows) are hyperintense on DWI (upper images) and the corresponding ADC maps (lower images) shows marked restricted diffusion for (a) right parotid $\mathrm{PA}$, (b) left parotid $\mathrm{WT}$, and (c) right parotid lymphoma cases. The mean $\mathrm{ADC}$ values of the lesions were $2.190 \times 10^{-3} \mathrm{~mm}^{2} / \mathrm{s}, 0.889 \times 10^{-3} \mathrm{~mm}^{2} / \mathrm{s}$, and $0.600 \times 10^{-3} \mathrm{~mm}^{2} / \mathrm{s}$, respectively

and MT. The sensitivity and specificity values for distinguishing PA from WT were $97 \%$ and $85 \%$, respectively, when the $\mathrm{ADC}$ cutoff value was 1.25 ; and $77 \%$ and $83 \%$, respectively, for the differentiation of PA from MT when the ADC cutoff value was 1.35. The conventional MRI findings of PA in one patient are shown in Figure 2. DWI and the corresponding ADC maps for PA, WT and a case of lymphoma are shown in Figure 3.

\section{Discussion}

The objective of this study was to investigate whether ADC values in DWI, as practical features of MRI, can contribute to preoperative histopathological differentiation in a clinical series. These results show that ADC is invaluable for differentiating PA, the most common parotid mass, from other histopathologies, rather than distinguishing malignant-benign lesions. When the ADC map was used as an independent predictor in the differentiation of PA from WT and MT, the respective AUC values were 0.948 and 0.927 in the ROC analysis. It can be concluded from these results that the $\mathrm{ADC}$ value can be accepted as a complementary tool to the clinical history, conventional MRI features and FNAB for the preoperative prediction of histopathology.
The main treatment modality for parotid masses is surgery. Therefore, obtaining the maximum information about the histopathology preoperatively strengthens the decision-making capability of the surgeon in respect of the extent of the surgery and informing the patient. As a regular approach to a disease, the epidemiology of parotid lesions, patient history and demographic data will provide information about the pathology of parotid lesions. Clinical features such as rigidity, rapid growth, pain and facial paralysis suggest a malignant lesion. Patient age can also add some information about the histopathology, as Warthin's tumor and malignant tumors are seen at a more advanced age, whereas pleomorphic adenoma is seen at a younger age (2). In the presented study, it was also found that PA was seen at younger ages $(p=0.001)$ with the median age of $44 \pm 21,55 \pm 10$ and $62 \pm 20$ years, respectively, in the PA, WT and MT groups. For the last decade, ultrasound with ultrasound-guided fine needle aspiration has been the first-line standard imaging modality. This method has an overall good accuracy rate (approximately $95 \%$ ) in cases where evaluation is made by an experienced cytologist $(5,6)$. There are, however, some limitations about FNAB because it is an invasive procedure (7). It may also give false negative and false positive results as it is operator and cytologist-dependent. Therefore, definitive results cannot be obtained. 
Currently, with increased accessibility to MRI and advancements in technology, the use of MRI in preoperative evaluation has become prevalent and some studies have claimed that MRI alone, without FNAB, can be sufficient to manage parotid lesions $(11,15)$. Therefore, even the findings of conventional MRI techniques contribute to the differentiation of whether the lesion histopathology is malignant or benign. The findings of border features (well defined or irregular), signal intensity on T2-weighted images (hyperintense or hypointense), deep lobe involvement, necrotic components, and the presence of lymphadenopathy presence will provide the surgeon with an idea before surgery $(4,16,17)$. However, conventional MRI findings are not sufficient, as the sensitivity and specificity of conventional MRI findings alone have been reported as $40 \%$ and $88 \%$, respectively (15). Nevertheless, conventional MRI features for pleomorphic adenoma histopathology, such as smooth surface and hypointensity on T1-weighted images and hyperintensity on T2-weighted images are characteristic in contrast to the controversial results for WT and MT on T2-weighted images $(4,11,18)$. The findings of our study were in accordance with the literature, as PA demonstrated $83 \%$ hyperintensity, and WT and MT demonstrated $81 \%$ and $47 \%$ hypointense signaling on T2-weighted images, respectively.

At the same time, especially adding DWI findings to conventional findings of MRI increases the sensitivity and specificity to $70 \%$ and $93 \%$, respectively (15). Furthermore, some studies have investigated more detailed features such as measuring the enhancement and washout patterns, and time signal intensity curves for each tumor and have found that time to peak is related to the microvessel extent and the washout ratio reflects the stromal cellularity level (18). In such studies, PA characteristically showed a curve with a gradual increase followed by a plateau. WT and MT showed similar curves of a steep rise followed by a plateau. In these dynamic MR studies, accuracy has been shown to be higher for WT (91.4\%-97.9\%), followed by MT (81.3-97.7\%) and PA (77.4-93\%) (19). Rather than the time signal intensity curves method, the ADC pattern in dynamic MRI is commonly used and is easier to evaluate without the need for a contrast agent $(11,17)$. Previous studies have focused on this, but the results are debatable as some have concluded that it is possible to differentiate benign and malignant tumors via ADC values whereas others have not $(9,12,14)$. Recent studies have revealed that on the basis of DWI alone, PA could be distinguished from malignant lesions whereas Warthin's tumor could not because of an overlap with malignant lesion ADC values (20). In some studies, myoepithelial adenomas have been shown to have ADC values similar to those of PA (13), but this is not so important because both lesions are benign and the extent of their surgeries are similar. However, the risk of progression of PA to malignancy is higher than that of myoepithelial adenomas, so awareness of recurrence should be higher. The results of our study showed that PA can be accurately differentiated from malignant and Warthin's tumors and ADC is useful for the most common histopathology differentiation in parotid lesions. The ADC level in lymphoma was seen to be similar to the levels reported in previous studies, which were low compared to the histopathology of other malignancies (14). This could be a topic of specific interest to be investigated in further studies to enable avoidance of unnecessary surgery $(10,17,21)$.

Using ADC values to differentiate the histopathology of parotid lesions is very important because the most commonly seen type of parotid tumor is $\mathrm{PA}$, and with the important diagnostic information obtained from MRI, there is the possibility of preventing unnecessary biopsies in majority of the parotid tumors.

\section{Conclusion}

Combining the quantitative values obtained from ADC measurements on DWI with clinical history and conventional MRI findings will add extra information for the differentiation of PA from both WT and MT. This can be considered a substantial complementary tool for the prediction of the histopathology in preoperative planning for parotid tumors. Despite these promising results, the use of MRI to detect the exact histopathology in parotid tumors is still in its infancy and more data is needed with larger series.

Ethics Committee Approval: Ethics committee approval was received for this study from the Clinical Research Ethics Committee of University of Health Sciences, Ankara Numune Training and Research Hospital (Approval Date: September 5, 2019; Approval Number: E1047-2019).

Informed Consent: Informed consent was obtained from the patients who participated in this study.

Peer-review: Externally peer-reviewed.

Author Contributions: Concept - F.C.E., K.M.Ö., D.S.C.; Design - F.C.E., K.M.Ö., D.S.C.; Supervision - K.M.Ö., D.S.C.; Materials - F.C.E., M.Ç., M.F.K., S.E.K.; Data Collection and/or Processing F.C.E., M.Ç., M. Çavuşoğlu, M.F.K.; Analysis and/or Interpretation - F.C.E., S.E.K., M. Çavuşoğlu; Literature Search - F.C.E., M.Ç., M.F.K., S.E.K.; Writing - F.C.E., M.Ç.; Critical Reviews - K.M.Ö., D.S.C., M. Çavuşoğlu.

Conflict of Interest: The authors have no conflicts of interest to declare.

Financial Disclosure: The authors declared that this study has received no financial support.

\section{References}

1. Barnes L, Eveson JW, Reichart P, Sidransky D, editors. World Health Organization classification of tumours. Pathology and genetics of the head and neck tumours. Lyon: IARC Press; 2005.p.209-81.

2. Lobo R, Hawk J, Srinivasan A. A review of salivary gland malignancies: common histologic types, anatomic considerations, and imaging strategies. Neuroimaging Clin N Am 2018; 28: 171-82. [Crossref]

3. Wang CW, Chu YH, Chiu DY, Shin N, Hsu HH, Lee JC, et al. JOURNAL CLUB: The Warthin tumor score: a simple and reliable method to distinguish Warthin tumors from pleomorphic adenomas and carcinomas. Am J Roentgenol 2018; 210: 1330-7. [Crossref] 
4. Christe A, Waldherr C, Hallett R, Zbaeren P, Thoeny H. MR imaging of parotid tumors: typical lesion characteristics in MR imaging improve discrimination between benign and malignant disease. Am J Neuroradiol 2011; 32: 1202-7. [Crossref]

5. Seethala RR, LiVolsi VA, Baloch ZW. Relative accuracy of fine-needle aspiration and frozen section in the diagnosis of lesions of the parotid gland. Head Neck 2005; 27: 217-23. [Crossref]

6. Gudmundsson JK, Ajan A, Abtahi J. The accuracy of fine-needle aspiration cytology for diagnosis of parotid gland masses: a clinicopathological study of 114 patients. J Appl Oral Sci 2016; 24: 561-7. [Crossref]

7. Supriya M, Denholm S, Palmer T. Seeding of tumor cells after fine needle aspiration cytology in benign parotid tumor: a case report and literature review. Laryngoscope 2008; 118: 263-5. [Crossref]

8. Koyuncu M, Şeşen T, Akan H, Ismailoglu AA, Tanyeri Y, Tekat A, et al. Comparison of computed tomography and magnetic resonance imaging in the diagnosis of parotid tumors. Otolaryngol Head Neck Surg 2003; 129: 726-32. [Crossref]

9. Matsushima N, Maeda M, Takamura M, Takeda K. Apparent diffusion coefficients of benign and malignant salivary gland tumors. Comparison to histopathological findings. J Neuroradiol 2007; 34 : 183-9. [Crossref]

10. Eida S, Sumi M, Nakamura T. Multiparametric magnetic resonance imaging for the differentiation between benign and malignant salivary gland tumors. J Magn Reson Imaging 2010; 31: 6739. [Crossref]

11. Karaman CZ, Tanyeri A, Ozgur R. Parotid gland tumors: comparison of conventional and diffusion-weighted MR imaging findings with histopathological results. Turk J Radiol 2018; 36: 60-8. [Crossref]

12. Eida S, Sumi M, Sakihama N, Takahashi H, Nakamura T. Apparent diffusion coefficient mapping of salivary gland tumors: prediction of the benignancy and malignancy. Am J Neuroradiol 2007; 28: 116-21.

13. Habermann CR, Arndt C, Graessner J, Diestel L, Petersen KU, Reitmeier F, et al. Diffusion-weighted echo-planar MR imag- ing of primary parotid gland tumors: is a prediction of different histologic subtypes possible? Am J Neuroradiol 2009; 30: 591-6. [Crossref]

14. Munhoz L, dos Anjos Ramos EA, Im DC, Hisatomi M, Yanagi $Y$, Asaumi J, et al. Application of diffusion-weighted magnetic resonance imaging in the diagnosis of salivary gland diseases: a systematic review. Oral Surg Oral Med Oral Pathol Oral Radiol 2019; 128: 280-310. [Crossref]

15. Fassnacht W, Schmitz S, Weynand B, Marbaix E, Duprez T, Hamoir M. Pitfalls in preoperative work-up of parotid gland tumours: 10-year series. B-ENT 2013; 9: 83-8.

16. Prades JM, Oletski A, Faye MB, Dumollard JM, Timoshenko AP, Veyret C, et al. [Parotid gland masses: diagnostic value of MR imaging with histopathologic correlations.] Morphologie 2007; 91: 44-51. [Crossref]

17. Elmokadem AH, Abdel Khalek AM, Abdel Wahab RM, Tharwat N, Gaballa GM, Elata MA, et al. Diagnostic accuracy of multiparametric magnetic resonance imaging for differentiation between parotid neoplasms. Can Assoc Radiol J 2019; 70: 26472. [Crossref]

18. Alibek S, Zenk J, Bozzato A, Lell M, Grunewald M, Anders K, et al. The value of dynamic MRI studies in parotid tumors. Acad Radiol 2007; 14: 701-10. [Crossref]

19. Ogawa T, Kojima I, Ishii R, Sakamoto M, Murata T, Suzuki T, et al. Clinical utility of dynamic-enhanced MRI in salivary gland tumors: retrospective study and literature review. Eur Arch Otorhinolaryngol 2018; 275: 1613-21. [Crossref]

20. Matsusue E, Fujihara Y, Matsuda E, Tokuyasu Y, Nakamoto S, Nakamura K, et al. Differentiating parotid tumors by quantitative signal intensity evaluation on MR imaging. Clin Imaging 2017; 46: 37-43. [Crossref]

21. Razek AAKA, Samir S, Ashmalla GA. Characterization of parotid tumors with dynamic susceptibility contrast perfusion-weighted magnetic resonance imaging and diffusion-weighted MR imaging. J Comput Assist Tomogr 2017; 41: 131-6. [Crossref] 